\title{
RECURSIVE COMBINATORIAL ASPECTS OF COMPACTIFIED MODULI SPACES
}

\author{
LUCIA CAPORASO
}

\section{Contents}

1. Introduction

2. Compactified Jacobians and Néron models

2.1. Jacobians, Picard schemes and Néron models 2

2.2. Combinatorics of Néron models 3

2.3. Compactifying Néron models 4

3. Moduli of curves: tropicalization and analytification 6

3.1. Moduli spaces of algebraic and tropical curves 6

3.2. Skeleta and tropicalizations 7

3.3. Algebraic and tropical admissible covers 9

4. Compactified Jacobians 10

4.1. Compactifying Jacobians over $\bar{M}_{g} \quad 10$

4.2. Compactified Jacobians in degree $g-1 \quad 11$

4.3. Jacobians in degree $g$ : Néron compactified Jacobians 13

References 15

\section{INTRODUCTION}

In recent years an interesting connection has been established between some moduli spaces of algebro-geometric objects (e.g. algebraic stable curves) and some moduli spaces of polyhedral objects (e.g. tropical curves).

In loose words, this connection expresses the Berkovich skeleton of a given algebro-geometric moduli space as the moduli space of the skeleta of the objects parametrized by the given space; it has been proved to hold in two important cases: the moduli space of stable curves and the moduli space of admissible covers. Partial results are known in other cases.

This connection relies on the study of the boundary of the algebro-geometric moduli spaces and on its recursive, combinatorial properties, some of which have been long known and are now viewed from a new perspective.

We will describe some results in this area by focusing on the moduli spaces of curves, line bundles on curves (i.e. Jacobians), and coverings of curves. As we said, it was clear for a long time that combinatorial aspects play a

Date: January 8, 2018. 
significant role in compactifying moduli spaces. A notable example is the structure of the Néron model of the Jacobian of a curve. Of course, Néron models are not compact, but they are a first step towards compactifying Jacobians. We begin the paper by describing Néron models, their combinatorial properties, and the recursive structure of their compactification. Then we turn to moduli spaces of curves and illustrate the connection introduced at the beginning. We return to compactified Jacobians in the last section and describe some recent partial results.

\section{Compactified Jacobians and Néron models}

2.1. Jacobians, Picard schemes and Néron models. Let $X$ be a connected, reduced, projective curve of genus $g$ over an algebraically closed field $k$. The set of isomorphism classes of line bundles of degree 0 on every irreducible component of $X$ is an algebraic group of dimension $g$, denoted by $J_{X}$, and called the Jacobian of $X$. More exactly, $J_{X}$ is the moduli space for line bundles of multidegree $(0,0, \ldots, 0)$ on $X$.

$J_{X}$ is projective, hence an abelian variety, if $X$ is smooth but not if $X$ is singular (with exceptions). Constructing compactifications for $J_{X}$ is a classical problem which can be stated as follows. Let

$$
X \hookrightarrow \mathcal{X} \stackrel{f}{\longrightarrow} \operatorname{Spec} R
$$

be a family of curves over the spectrum of a discrete valuation ring $R$, with $X$ as special fiber. Assume the generic fiber, $\mathcal{X}_{K}$, to be a nonsingular curve over the quotient field, $K$, of $R$, and let $J_{\mathcal{X}_{K}} \rightarrow$ Spec $K$ be its Jacobian. The problem is to find "good" models of $J_{\mathcal{X}_{K}}$ over $\operatorname{Spec} R$, where "good" means: (a) projective, (b) with a moduli interpretation, (c) such that the special fiber depends only on $X$ and not on the family $f$. We shall refer to such models, and to their special fiber, as compactified Jacobians.

We shall begin by looking for models that satisfy (b) and (c) together with the weaker requirement of being separated, rather than projective.

A natural model of $J_{\mathcal{X}_{K}}$ is the relative Jacobian (a group scheme over $R$ )

$$
J_{\mathcal{X} / R} \longrightarrow \operatorname{Spec} R \text {. }
$$

This is a separated model for $J_{\mathcal{X}_{K}}$, but it does not have satisfactory moduli properties. Indeed, suppose we have a line bundle $\mathcal{L}$ over $\mathcal{X}$ having relative degree 0 on the fibers of $f$. Then we have a moduli morphism $\mu_{\mathcal{L}_{K}}: \operatorname{Spec} K \rightarrow J_{\mathcal{X}_{K}}$ whose image corresponds to the restriction of $\mathcal{L}$ to $\mathcal{X}_{K}$. For a model to have good moduli properties we want the map $\mu_{\mathcal{L}_{K}}$ to extend to a morphism from $\operatorname{Spec} R$ to the model (so that the image of the special point is determined by the restriction of $\mathcal{L}$ to the special fiber). For the relative Jacobian this requirement easily fails.

Another natural model is the relative degree-0 Picard scheme

$$
\operatorname{Pic}_{\mathcal{X} / R}^{0} \longrightarrow \operatorname{Spec} R \text {. }
$$


Now, this has a good moduli interpretation, as any moduli map $\mu_{\mathcal{L}_{K}}$ will certainly extend to a map $\mu_{R}: \operatorname{Spec} R \rightarrow \operatorname{Pic}_{\mathcal{X} / R}^{0}$, and $\mu_{R}$ is itself a moduli map. The problem now is that the extension $\mu_{R}$ may fail to be unique, that is, (2) is not a separated morphism, in general.

We have thus two natural models, one separated with bad moduli properties, the other with good moduli properties but not separated. Does there exist a compromise with better behaviour than both of them? An answer to this question comes from the theory of Néron models.

Our moduli requirement above (i.e. the existence of a unique extension for maps of the form $\mu_{\mathcal{L}_{K}}$ ) is a special case of the mapping property satisfied by Néron models, whose existence has been established in [29]. We state the following special case of Néron's famous theorem, using the above notation.

Theorem 2.1.1 (Néron). There exists a smooth and separated group scheme of finite type

$$
N\left(J_{\mathcal{X}_{K}}\right) \longrightarrow \operatorname{Spec} R
$$

whose generic fiber is $J_{\mathcal{X}_{K}}$, satisfying the following mapping property:

Let $Y_{R} \rightarrow \operatorname{Spec} R$ be smooth, $Y_{K}$ its generic fiber, and $\mu_{K}: Y_{K} \rightarrow J_{\mathcal{X}_{K}} a$ morphism. Then $\mu_{K}$ extends uniquely to a morphism $\mu_{R}: Y_{R} \rightarrow N\left(J_{\mathcal{X}_{K}}\right)$.

Remark 2.1.2. The Néron model does not commute with ramified base change, therefore it is a separated model for $J_{\mathcal{X}_{R}}$ with good mapping properties, but not functorial ones.

The relation of the Néron model with the relative Jacobian and the relative Picard scheme has ben established by Raynaud who proved, in [32, that $N\left(J_{\mathcal{X}_{K}}\right) \rightarrow \operatorname{Spec} R$ is the maximal separated quotient of $\operatorname{Pic}_{\mathcal{X} / R}^{0} \rightarrow \operatorname{Spec} R$.

2.2. Combinatorics of Néron models. We shall turn to the geometric structure of our Néron models. We assume from now on that the curve $X$ is nodal, write $X=\cup_{v \in V} C_{v}$ for its irreducible components, and consider the dual graph, $G_{X}$, of $X$ :

$$
G_{X}:=(V, E=\operatorname{Sing}(X), V \stackrel{h}{\longrightarrow} \mathbb{Z})
$$

with $h(v)=g\left(C_{v}\right)$, where $g\left(C_{v}\right)$ is the genus of the normalization, $C_{v}^{\nu}$. The genus of $G_{X}$ is the same as the arithmetic genus of $X$, i.e.

$$
g(X)=g\left(G_{X}\right)=b_{1}\left(G_{X}\right)+\sum_{v \in V} h(v) .
$$

It is well known that $J_{X}$ fits into an exact sequence of algebraic groups:

$$
0 \longrightarrow\left(k^{*}\right)^{b_{1}\left(G_{X}\right)} \longrightarrow J_{X} \longrightarrow \prod_{v \in V} J_{C_{v}^{\nu}} \longrightarrow 0 .
$$

Denote by $N_{X}$ the special fiber of $N\left(J_{\mathcal{X}_{K}}\right) \rightarrow$ Spec $R$. We shall state some results of Raynaud, 32], and Oda-Seshadri, 30, which establish that $N_{X}$ is a disjoint union of copies of the Jacobian of $X$ indexed by a combinatorial invariant of the curve. 
To do that we need some combinatorial preliminaries. Fix an orientation on $G=G_{X}$ (whose choice is irrelevant), let $C_{0}(G, \mathbb{Z})$ and $C_{1}(G, \mathbb{Z})$ be the standard groups of $i$-chains, generated over $\mathbb{Z}$ by $V$ if $i=0$, and by $E$ if $i=1$. Next, let $\partial: C_{1}(G, \mathbb{Z}) \rightarrow C_{0}(G, \mathbb{Z})$ be the usual boundary (mapping an edge $e$ oriented from $u$ to $v$ to $u-v)$, and $\delta: C_{0}(G, \mathbb{Z}) \rightarrow C_{1}(G, \mathbb{Z})$ the coboundary (mapping a vertex $v$ to $\sum e_{v}^{+}-\sum e_{v}^{-}$where the first sum is over all edges originating from $v$ and the second over all edges ending at $v$ ).

Now we can state the following.

Proposition 2.2.1 (Raynaud, Oda-Seshadri). Let $\mathcal{X} \rightarrow$ Spec $R$ have nodal special fiber and regular total space. Then the special fiber, $N_{X}$, of the Néron model $N\left(J_{\mathcal{X}_{K}}\right) \longrightarrow \operatorname{Spec} R$ is a union of copies of $J_{X}$ as follows

$$
N_{X} \cong \sqcup_{i \in \Phi_{X}}\left(J_{X}\right)_{i}
$$

where $\Phi_{X}$ is the following finite group

$$
\Phi_{G} \cong \frac{\partial \delta C_{0}(G, \mathbb{Z})}{\partial C_{1}(G \mathbb{Z})}
$$

In particular, the number of irreducible components of $N_{X}$ equals the number of spanning trees of $G_{X}$.

The last claim follows from Kirchhoff-Trent, or Kirchhoff matrix,Theorem.

2.3. Compactifying Néron models. From now on we apply the notation introduced in Proposition 2.2.1 and for any connected nodal curve $X$ we denote by $N_{X}$ the special fiber of the Néron model of the Jacobian associated to a family $\mathcal{X} \rightarrow \operatorname{Spec} R$ with $\mathcal{X}$ regular.

If $X$ is a singular curve with $b_{1}\left(G_{X}\right) \neq 0$ (i.e. $X$ not of "compact type"), then the Néron model $N\left(J_{\mathcal{X}_{K}}\right) \rightarrow$ Spec $R$ is not projective, as its special fiber is not projective by the exact sequence (4).

Now, the Picard scheme has good moduli properties and the Néron model is its maximal separated quotient. We introduce a terminology to distinguish compactified Jacobians which also compactify the Néron model.

A compactified Jacobian $\bar{P} \longrightarrow$ Spec $R$ is said to be of Néron type (or a Néron compactified Jacobian) if its special fiber, written $\bar{P}_{X}$, contains $N_{X}$ as a dense open subset. We shall also say that $\bar{P}_{X}$ is of Néron type.

As we shall see, Néron compactified Jacobians do exist, but there exist also interesting compactified Jacobians not of Néron type.

The notion originates from [30] and [12, although the terminology was introduced later, in [14. Oda and Seshadri, in [30, treated the case of a fixed singular curve $X$ (rather than a family of curves), and constructed compactified Jacobians in this less general sense. They nonetheless established the link with Néron models and constructed compactified Jacobians whose smooth locus is isomorphic to $N_{X}$. This was extended to families of curves later. In 11 and 12 a class of compactified Jacobians of Néron type was proved to exist and to form a family over the moduli space of stable 
curves, $\overline{\mathcal{M}_{g}}$. Such families, denoted by $\psi_{d}: \overline{\mathcal{P}}_{g}^{d} \longrightarrow \overline{\mathcal{M}_{g}}$, are indexed by the integers $d$ such that

$$
(d-g+1,2 g-2)=1 \text {. }
$$

For any stable curve $X$ the fiber of $\psi_{d}$ over $X$, written $\bar{P}_{X}^{d}$, contains $N_{X}$ as a dense open subset equal to its smooth locus, and it is thus of Néron type.

In a similar vein, in [26] and in [25] other Néron compactified Jacobians were found among the ones constructed by Esteves in [19], and called "fine" compactified Jacobians. The word "fine" is quite appropriate, as all known Néron compactified Jacobians are as fine a moduli space as they can be, i.e. they admit a universal (or "Poincaré") line bundle.

We now show how Néron Jacobians are recursive compactifications of Néron models. First, for a connected graph $G$, we introduce the following

$$
\mathcal{C}(G):=\{S \subset E(G): G-S \text { is connected }\},
$$

with partial order given by reverse inclusion. The maximal element of $\mathcal{C}(G)$ is $\emptyset$, and the minimal elements are the $S \subset E$ such that $G-S$ is a spanning tree. Moreover, $\mathcal{C}(G)$ is a graded poset with respect to the rank function $S \mapsto g(G-S)$.

If $G$ is the dual graph of the curve $X$ then $S \in \mathcal{C}(G)$ is a set of nodes of $X$. We denote by $X_{S}^{\nu}$ the desingularization of $X$ at $S$, so that $X_{S}^{\nu}$ is a connected nodal curve of genus $g(G-S)$. Recall that $N_{X_{S}^{\nu}}$ denotes the special fiber of the Néron model of its Jacobian. The following follows from [12, Thm. 7.9].

Theorem 2.3.1. Let $\bar{P}_{X}^{d}$ be a Néron compactified Jacobian. Then

$$
\bar{P}_{X}^{d}=\bigsqcup_{S \in \mathcal{C}(G)} N_{S}
$$

with $N_{S} \cong N_{X_{S}^{\nu}}$ for every $S \in \mathcal{C}(G)$. Moreover (7) is a graded stratification, i.e. the following hold.

(1) $N_{S} \cap \overline{N_{S^{\prime}}} \neq \emptyset \Leftrightarrow N_{S} \subset \overline{N_{S^{\prime}}} \Leftrightarrow S^{\prime} \geq S$.

(2) $N_{S}$ is locally closed of pure dimension $g(G-S)$.

(3) The following is a rank-function on $\mathcal{C}(G)$

$$
\mathcal{C}(G) \longrightarrow \mathbb{Z} ; \quad S \mapsto \operatorname{dim} N_{S}
$$

Remark 2.3.2. The set of strata of minimal dimension in (7) are Néron models of curves whose dual graph is a spanning tree of $G_{X}$, hence they are irreducible. By Proposition 2.2.1, the number of such strata is equal to the number of irreducible components of $\bar{P}_{X}^{d}$.

If $X$ is not of compact type the strata of (7) are not all connected. Hence one naturally asks whether the stratification can be refined so as to have connected strata. We will answer this question later in the paper.

Remark 2.3.3. The theorem exhibits the compactification of the Néron model of $X$ in terms of the Néron models of partial normalizations of $X$. 
This phenomenon is an instance of what seems to be a widespread recursive behaviour for compactified moduli spaces. Namely, to compactify a space (e.g. $N_{X}$ ) one adds at the boundary the analogous spaces associated to simpler objects (e.g. $N_{X^{\prime}}$ with $X^{\prime}$ a connected partial normalization of $X$ ). Other examples of this recursive pattern will appear later in the paper.

The concept of "graded stratification" used in the Theorem will appear again, so we now define it in general.

A graded stratification of an algebraic variety, or a stack, $M$ by a poset $\mathcal{P}$ is a partition $M=\bigsqcup_{p \in \mathcal{P}} M_{p}$ such that the following hold for every $p, p^{\prime} \in \mathcal{P}$.

(1) $M_{p} \cap \overline{M_{p^{\prime}}} \neq \emptyset \Leftrightarrow M_{p} \subset \overline{M_{p^{\prime}}} \Leftrightarrow p^{\prime} \geq p$.

(2) $M_{p}$ is equidimensional and locally closed.

(3) The map from $\mathcal{P}$ to $\mathbb{N}$ sending $p$ to $\operatorname{dim} M_{p}$ is a rank function on $\mathcal{P}$.

\section{Moduli of CuRves: tropicalization And ANALYTIFICATION}

3.1. Moduli spaces of algebraic and tropical curves. Let $\mathcal{M}_{g, n}$ be the moduli space of smooth curves of genus $g$ with $n$ marked points. Assume $2 g-2+n>0$ so that $\mathcal{M}_{g, n}$ is never empty. It is well known that $\mathcal{M}_{g, n}$ is not projective (unless $g=0, n=3$ ) and is compactified by the moduli space of Deligne-Mumford stable curves, $\overline{\mathcal{M}}_{g, n}$; see [18, [22], 23], 20].

We will describe in (9) a stratification of $\overline{\mathcal{M}}_{g, n}$ which is recursive in the sense of Remark 2.3.3, that is, the boundary strata are expressed in terms of simpler moduli spaces $\mathcal{M}_{g^{\prime}, n^{\prime}}$. In this case "simpler"means $g \leq g^{\prime}$ and of smaller dimension, i.e. $n^{\prime}<n+3\left(g-g^{\prime}\right)$. These boundary strata will be described in (8).

Let $\mathcal{S G}_{g, n}$ be the poset of stable graphs of genus $g$ with $n$ legs, with the following partial order:

$$
G_{2} \geq G_{1} \quad \text { if } \quad G_{2}=G_{1} / S \quad \text { for some } \quad S \subset E\left(G_{1}\right)
$$

where $G_{1} / S$ is the graph obtained by contracting every edge of $S$ to a vertex. By the very definition (introduced in [10]), edge-contraction preserves $g$ and $n$, and it is easily seen to preserve stability.

To a stable curve, $X$, of genus $g$ with $n$ legs there corresponds a dual graph $G_{X} \in \mathcal{S G}_{g, n}$. With respect to what we defined in (3) the only new piece of data are the legs of $G_{X}$ which correspond to the $n$ marked points. Recall that the weight, $h(v)$, of a vertex $v$ is the geometric genus of the corresponding component of $X$. We denote by $\operatorname{deg}(v)$ the degree (or valency) of $v$.

For $G \in \mathcal{S G}_{g, n}$ we denote by $\mathcal{M}_{G}$ the moduli stack of curves whose dual graph is isomorphic to $G$. We have (see [1, Prop. 3.4.1])

$$
\mathcal{M}_{G}=\left[\left(\prod_{v \in V(G)} \mathcal{M}_{h(v), \operatorname{deg}(v)}\right) / \operatorname{Aut}(G)\right] .
$$

With this notation and the terminology at the end of Section 2 we state: 
Proposition 3.1.1. The following is a graded stratification of $\overline{\mathcal{M}}_{g, n}$

$$
\overline{\mathcal{M}}_{g, n}=\bigsqcup_{G \in \mathcal{S G}_{g}} \mathcal{M}_{G}
$$

One goal of the above descriptive result (whose proof, in [13], is not hard thanks to our consolidated knowledge of $\overline{\mathcal{M}_{g}}$ ) is to highlight the similarities of $\overline{\mathcal{M}}_{g, n}$ with the moduli space of extended (abstract) tropical curves, $\bar{M}_{g, n}^{\text {trop }}$, as we are going to show. First of all, an extended tropical curve is a metric graph, i.e. a graph $G$ whose edges are assigned a length, $\ell: E \rightarrow \mathbb{R}_{>0} \cup\{\infty\}$. We denote a tropical curve as follows

$$
\Gamma=(G ; \ell)=(V, E, w ; \ell) .
$$

The genus of $\Gamma$ is the genus of $G$. The word "extended" refers to the fact that we include edges of infinite length. In fact, abstract tropical curves are originally defined (in [28] and [10]) as compact spaces, so their edges have finite length. We allow edges of infinite lengths to obtain a compact moduli space which is the "tropicalization" of the moduli space of stable curves.

An extended tropical curve with $n$ marked points is a metric graph as above with the addition of a set of legs of the underlying graph.

There is a natural equivalence relation on tropical curves such that in every equivalence class there is a unique (up to isomorphism) curve whose underlying graph is stable.

The moduli space $\bar{M}_{g, n}^{\text {trop }}$ parametrizes extended tropical curves of genus $g$ with $n$ marked points up to this equivalence relation. Its first construction is due to Mikhalkin, for $g=0$, and to Brannetti-Melo-Viviani for compact tropical curves in any genus. The following statement summarises results from [27, [10] and [13].

Theorem 3.1.2. The moduli space of extended tropical curves, $\bar{M}_{g, n}^{\mathrm{trop}}$, is a compact and normal topological space of dimension $3 g-3+n$. It admits a graded stratification

$$
\bar{M}_{g, n}^{\text {trop }}=\bigsqcup_{G \in \mathcal{S G}_{g, n}^{*}} \bar{M}_{G}^{\text {trop }}
$$

where $\bar{M}_{G}^{\text {trop }}$ is the locus of curves having $G$ as underlying graph and $\mathcal{S G}_{g, n}^{*}$ is the poset dual to $\mathcal{S G}_{g, n}$ (i.e. with reverse partial order).

3.2. Skeleta and tropicalizations. Proposition 3.1.1 and Theorem 3.1.2 show that $\bar{M}_{g, n}^{\text {trop }}$ has a graded stratification dual to that of $\overline{\mathcal{M}}_{g, n}$. Therefore we ask whether there exists some deeper relation between $\overline{\mathcal{M}}_{g, n}$ and $\bar{M}_{g, n}^{\text {trop }}$.

A positive answer can be given through the theory of analytifications of algebraic schemes developed in [9], and through its connections to tropical geometry; see 24 and 31. Let us introduce the space $\bar{M}_{g, n}^{\text {an }}$, the analytification of $\overline{\mathcal{M}}_{g, n}$ in the sense of Berkovich. Recall that a point in $\bar{M}_{g, n}^{\text {an }}$ corresponds, up to base change, to a stable curve over an algebraically closed 
field $K$ complete with respect to a non-archimedean valuation; as $\bar{M}_{g, n}$ is projective, this is the same as a stable curve over the ring of integers of $K$.

From the general theory (see also [33] and [1]) we have that for every space with a toroidal structure, like the stack $\overline{\mathcal{M}}_{g, n}$ (with toroidal structure given by its boundary), one associates the Berkovich skeleton which is a generalised, extended cone complex onto which the analytification retracts. We write $\bar{\Sigma}\left(\overline{\mathcal{M}}_{g, n}\right)$ for the Berkovich skeleton of $\overline{\mathcal{M}}_{g, n}$ and $\bar{M}_{g, n}^{\text {an }} \stackrel{\rho}{\longrightarrow} \bar{\Sigma}\left(\overline{\mathcal{M}}_{g, n}\right)$ for the retraction.

The connection between tropical geometry and Berkovich theory originates from the fact that Berkovich skeleta can be viewed as tropicalizations of algebraic varieties. As we are going to see, the picture for curves is quite clear, whereas other interesting situations (some treated later in the present paper) are still open to investigations; we refer to [21], for recent progress for higher dimensional varieties.

Now, tropical curves can be viewed as tropicalizations (or skeleta) of curves over algebraically closed, complete, non-archimedean fields. This is clarified by the following statement, combining results of [35, 34] [6], 5] (for the first part) and of [1] (for the second part).

Theorem 3.2.1. There exists a tropicalization map

$$
\text { trop }: \bar{M}_{g, n}^{\text {an }} \rightarrow \bar{M}_{g, n}^{\text {trop }}
$$

that sends the class of a stable curve over the (algebraically closed, complete, non-archimedean) field $K$ to its skeleton.

There is a natural isomorphism, $\bar{\Sigma}\left(\overline{\mathcal{M}}_{g, n}\right) \cong \bar{M}_{g, n}^{\text {trop }}$, through which the above map factors as follows

$$
\text { trop : } \bar{M}_{g, n}^{\text {an }} \stackrel{\rho}{\longrightarrow} \bar{\Sigma}\left(\overline{\mathcal{M}}_{g, n}\right) \stackrel{\cong}{\longrightarrow} \bar{M}_{g, n}^{\text {trop }} .
$$

We need to define the tropicalization map and explain the word "skeleton". As we said, a point in $\bar{M}_{q, n}^{\text {an }}$ is a class of stable curves, $\mathcal{X} \rightarrow \operatorname{Spec} R$, where $R$ is the valuation ring of $K$; let $X$ be its special fiber. Then the image of this point via the map trop is the tropical curve $(G ; \ell)$, where $G$ is the dual graph of $X$ and, for every node $e \in E(G)$, the value $\ell(e)$ is determined by the local geometry of $\mathcal{X}$ at $e$ as measured by the given valuation. Such a tropical curve $(G ; \ell)$ is called the skeleton, of the stable curve $\mathcal{X} \rightarrow \operatorname{Spec} R$ or of the stable curve $\mathcal{X}_{K}$ over $K$.

Concluding in loose words: the skeleton of $\overline{\mathcal{M}}_{g, n}$ is the moduli space of skeleta of stable curves.

The structure of the proof of the above theorem is such that it may apply to other situations. In fact it has been applied by Cavalieri-MarkwigRanganathan to another remarkable case, the compactification of the Hurwitz spaces, as we shall now explain. 
3.3. Algebraic and tropical admissible covers. Consider the moduli space of admissible covers, $\overline{\mathcal{H}}_{\bullet}$, which here (as in [16]), is the normal compactification of the classical space of Hurwitz covers $\mathcal{H}_{\bullet}$. We use the subscript "•" to simplify the notation needed to express the usual discrete invariants. Indeed, a more precise notation would be $\mathcal{H}_{\bullet}=\mathcal{H}_{g, h}(\underline{\pi})$ for the Hurwitz space parametrizing degree- $d$ covers of a smooth curve of genus $h$ by a smooth curve of genus $g$ with exactly $b$ branch points with ramification profile prescribed by a set of $b$ partitions of $d$, written $\underline{\pi}=\left(\pi_{1}, \ldots, \pi_{b}\right)$.

In [16] the authors define tropical admissible covers, construct their moduli space $\bar{H}_{\bullet}^{\text {trop }}$ and establish an analogue to Theorem 3.2.1. We shall now outline the procedure and give some details.

The first step is to associate a dual combinatorial entity to the algebrogeometric one. Indeed, one associates to an admissible cover a map of graphs, which we call the dual graph cover. The set of all dual graph covers is endowed with a poset structure by means of edge-contractions (similarly to the poset set of stable graphs). We denote by $\mathcal{A}_{\bullet}$ this poset, as its objects can be viewed as admissible maps of graphs. For any $\Theta \in \mathcal{A}_{\bullet}$ we denote by $\mathcal{H}_{\Theta}$ the locus in $\overline{\mathcal{H}}_{\bullet}$ of admissible covers whose dual graph map is $\Theta$.

The second step is to enrich the dual combinatorial entity with a tropical, or polyhedral, structure. In [16] tropical admissible covers are defined by metrizing, in a suitable way, dual maps of graphs. The moduli space of tropical admissible covers is denoted by $\bar{H}_{\bullet}^{\text {trop }}$, the bar over $H$ indicates that they are "extended", i.e. edge-lengths are allowed to be infinite. For $\Theta \in \mathcal{A}_{\text {• }}$ the stratum parametrizing tropical admissible covers having $\Theta$ as underlying graph map is denoted by $\bar{H}_{\Theta}^{\text {trop }}$ and shown to be the quotient of an extended real cone.

The third and last step is to use analytification and tropicalization to establish an explicit link between the algebraic and the tropical moduli space. Indeed, essentially by construction, we have dual stratifications

$$
\overline{\mathcal{H}}_{\bullet}=\bigsqcup_{\Theta \in \mathcal{A}_{\bullet}} \mathcal{H}_{\Theta} \quad \text { and } \quad \bar{H}_{\bullet}^{\text {trop }}=\bigsqcup_{\Theta \in \mathcal{A}_{\bullet}^{*}} \bar{H}_{\Theta}^{\text {trop }} .
$$

This duality can be read from the following Theorem.

Theorem 3.3.1 (Cavalieri-Markwig-Ranganathan). There is a tropicalization map trop : $\overline{\mathcal{H}}_{\bullet}^{\text {an }} \rightarrow \bar{H}_{\bullet}^{\text {trop }}$ which factors as follows

$$
\text { trop : } \overline{\mathcal{H}}_{\bullet}^{\text {an }} \stackrel{\rho}{\longrightarrow} \bar{\Sigma}\left(\overline{\mathcal{H}}_{\bullet}\right) \longrightarrow \bar{H}_{\bullet}^{\text {trop }} \text {. }
$$

The map $\overline{\mathcal{H}}_{\bullet}^{\text {an }} \stackrel{\rho}{\longrightarrow} \bar{\Sigma}\left(\overline{\mathcal{H}}_{\bullet}\right)$ is the retraction of $\overline{\mathcal{H}}_{\bullet}^{\text {an }}$ onto its Berkovich skeleton, as described in subsection 3.2. This result is compatible with the analogous one for $\overline{\mathcal{M}_{g}}$ through the canonical forgetful maps from $\overline{\mathcal{H}}_{\bullet}$ to the moduli spaces of stable curves; see [16, Thm. 4].

What about other moduli spaces? Are Theorems 3.2.1 and 3.3.1 part of some general picture where skeleta of algebraic moduli spaces (e.g. the skeleton of $\overline{\mathcal{M}}_{g, n}$ ) can be described as moduli spaces for combinatorial entities 
(e.g. tropical curves) which are skeleta of the objects (e.g. stable curves) parametrized by the algebraic moduli spaces? As we saw, the first step is to identify a suitable partially ordered set of combinatorial objects to associate to the algebro-geometric ones.

In the next section we shall look at the theory of compactified Jacobians from this point of view.

\section{Compactified Jacobians}

4.1. Compactifying Jacobians over $\bar{M}_{g}$. Let us go back to compactify Jacobians of curves and, with the discussion of the previous section in mind, approach the problem from the point of view of the moduli theory of stable curves. Consider the universal Jacobian over the moduli space of smooth curves and look for a compactification of it over $\overline{\mathcal{M}_{g}}$ satisfying the requirements we discussed earlier.

For reasons that will be clear later, it is convenient to extend our considerations to Jacobians of all degree. For a curve $X=\cup_{v \in V} C_{v}$ and any multidegree $\underline{d} \in \mathbb{Z}^{V}$ we write $\operatorname{Pic} \underline{d}(X)$ for the moduli space of line bundles of multidegree $\underline{d}$. Now, $J_{X}$ is identified with $\operatorname{Pic}^{(0, \ldots, 0)}(X)$ and we have non-canonical isomorphisms $J_{X} \cong \operatorname{Pic} \underline{d}(X)$.

The universal degree- $d$ Jacobian over $\mathcal{M}_{g}$ is a morphism $\mathcal{P}_{g}^{d} \rightarrow \mathcal{M}_{g}$, whose fiber over the point parametrizing a smooth curve $X$ is $\operatorname{Pic}^{d}(X)$. We want to construct a compactification of $\mathcal{P}_{g}^{d}$ over $\overline{\mathcal{M}_{g}}$ by a projective morphism $\overline{\mathcal{P}}_{g}^{d} \rightarrow \overline{\mathcal{M}_{g}}$ such that $\overline{\mathcal{P}}_{g}^{d}$ has a moduli description. We shall refer to such a space as a compactified universal degree- $d$ Jacobian.

From this perspective there is a natural approach to the problem, namely imitate the construction of $\overline{\mathcal{M}_{g}}$ itself. Recall that the moduli scheme, $\bar{M}_{g}$, of the stack $\overline{\mathcal{M}_{g}}$ was constructed by Gieseker in [20] as the GIT-quotient of the Hilbert scheme of $n$-canonically embedded curves $(n \gg 0)$. The moduli stack $\overline{\mathcal{M}_{g}}$ is the quotient stack associated to this quotient.

Now, as the Hilbert scheme of $n$-canonically embedded curves of genus $g$ has such a beautiful GIT-quotient, why shouldn't the Hilbert scheme of all projective curves of degree $d \gg 0$ and genus $g$ have a beautiful GIT-quotient? And why shouldn't this quotient be a candidate for a compactification of the universal degree- $d$ Jacobian? Indeed, this is what happens, and the GIT quotient of this Hilbert scheme is our compactified universal degree- $d$ Jacobian, $\bar{P}_{g}^{d}$. The corresponding quotient stack is denoted by $\overline{\mathcal{P}}_{g}^{d}$.

Now, as $d$ varies, the spaces $\overline{\mathcal{P}}_{g}^{d}$ are not isomorphic to one another, indeed their fibers over certain singular curves are not even birational to one another. Again, we see the phenomenon (appearing already in [30]) that non isomorphic compactifications of the Jacobian of a fixed singular curve exist. In the present case the various models depend on the degree $d$.

As for the basic properties of the spaces $\overline{\mathcal{P}}_{g}^{d}$ as $d$ varies, there is a special set of degrees, namely those such that (6) holds, such that $\bar{P}_{g}^{d}$ is a geometric 
GIT-quotient and has good moduli properties, so that its points correspond to geometric objects up to a certain equivalence relation. Moreover, the natural (projective) morphism $\psi_{d}: \overline{\mathcal{P}}_{g}^{d} \longrightarrow \overline{\mathcal{M}_{g}}$ is a strongly representable map of Deligne-Mumford stacks. As we said in subsection 2.3 in this case all fibers of $\psi_{d}$ are Néron compactified Jacobians.

Now, (6) fails if $d=g-1$ whereas it holds if $d=g$. We shall concentrate on these two cases, interesting for different reasons, and give a combinatorial analysis of the compactified Jacobian.

4.2. Compactified Jacobians in degree $g-1$. We begin by reviewing an idea of Beauville. Let us fix $g \geq 2$. Among degree- $d$ Jacobians, the case $d=g-1$ has been object of special interest for its strong connections with the Theta divisor, the Schottky problem, the Prym varieties; in particular, it has been studied in 8 .

Let us approach the problem of compactifying the degree $(g-1)$-Jacobian of a curve $X$ with $G=(V, E, h)$ as dual graph. We expect a good compactification to have finitely many irreducible components and each component to parametrize (at least away from the boundary) line bundles on $X$ of a fixed multidegree $\underline{d}$ such that $|\underline{d}|=g-1$. Now the question is to determine these "special" multidegrees. Consider the identity

$$
g-1=\sum_{v \in V}(h(v)-1)+|E|
$$

We can interpret the first term (i.e. the summation) as carrying the topological invariants of $X$, and the second term, $|E|$, as carrying the combinatorial ones. Now, while the first term exhibits the single contribution of each vertex/component, the second does not. So we may ask how to distribute the second term among the various vertices in a combinatorially meaningful way. A natural solution is to consider an orientation, $O$, on $G$, and denote by $\underline{t}_{v}^{O}$ the number of edges having $v$ as target. Then $\sum_{v \in V} \underline{t}_{v}^{O}=|E|$. Therefore, if we define a multidegree $\underline{d}^{O}$ as follows

$$
\underline{d}_{v}^{O}:=h(v)-1+\underline{t}_{v}^{O},
$$

for every $v \in V$, we have $\left|\underline{d}^{O}\right|=g-1$. As there are only finitely many orientations on a graph, by the above rule we have picked a finite set of special multidegrees of degree $g-1$. More precisely, it may happen that two orientations, $O$ and $O^{\prime}$, give the same multidedegree; in such a case we say that $O$ and $O^{\prime}$ are equivalent. We denote by $\bar{O}(G)$ the set of such equivalence classes of orientations on $G$. Now, a closer look reveals that $\bar{O}(G)$ is still too big for it to parametrize the irreducible components of a compactified Jacobian. Indeed, from the discussion in Subsection 2.2 we expect the number of such components to be at most equal to $\left|\Phi_{G}\right|$, whereas we have $|\bar{O}(G)|>\left|\Phi_{G}\right|$ in general (for example for a 2-cycle, as in the picture below). 
So, to compactify the degree- $(g-1)$ Jacobian we must distinguish a special type of orientations. These are called totally cyclic orientations, defined as orientations such that any two vertices in the same connected component of $G$ lie in a directed cycle. It tuns out that if two orientations are equivalent, one is totally cyclic if the other one is. The set of equivalence classes of totally cyclic orientations on $G$ is denoted by $\bar{O}^{0}(G)$.

In the picture below we have the four orientations on a 2-cycle. The first two are totally cyclic and equivalent. The last two are not totally cyclic.
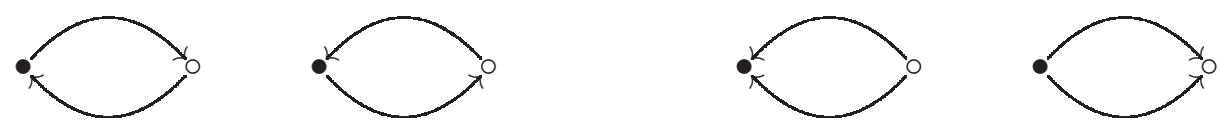

We shall adopt the convention that the empty orientation on the graph consisting of only one vertex and no edges is totally cyclic. We notice the following facts.

Remark 4.2.1. (a) $\bar{O}^{0}(G)$ is empty if and only if $G$ contains some bridge.

(b) Assume $G$ connected. Then $\left|\bar{O}^{0}(G)\right| \leq\left|\Phi_{G}\right|$ with equality if and only if $|V|=1$.

The set of all orientations on the spanning subgraphs of a graph $G$ admits a partial order as follows. Let $O_{S_{1}}$ and $O_{S_{2}}$ be two orientations on $G-S_{1}$ and $G-S_{2}$ respectively, where $S_{i} \subset E(G)$ for $i=1,2$. We set $O_{S_{1}} \leq O_{S_{2}}$ if $G-S_{1} \subset G-S_{2}$ and if the restriction of $O_{S_{2}}$ to $G-S_{1}$ equals $O_{S_{1}}$.

This definition is compatible with the equivalence relation defined above, and hence the set of all equivalence classes of totally cyclic orientations on $G$ is a poset, which we shall denote as follows

$$
\overline{\mathcal{O P}}^{0}(G):=\bigsqcup_{S \subset E(G)} \bar{O}^{0}(G-S)
$$

Finally, we are ready to exhibit a graded stratification of $\bar{P}_{X}^{g-1}$ governed by totally cyclic orientations, by rephrasing some results in [15].

Proposition 4.2.2. Let $X$ be a stable curve of genus $g$ and $G$ its dual graph. Then the following is a graded stratification

$$
\bar{P}_{X}^{g-1}=\bigsqcup_{\overline{O_{S}} \in \overline{\mathcal{O P}}^{0}(G)} P_{X}^{O_{S}},
$$

and we have natural isomorphisms for every $\overline{O_{S}} \in \overline{\mathcal{O P}}^{0}(G)$

$$
P_{X}^{O_{S}} \cong \operatorname{Pic}^{\underline{d}^{O}}\left(X_{S}^{\nu}\right)
$$


The isomorphisms (12) exhibit the recursive behaviour described in Remark 2.3.3. Indeed, $\operatorname{Pic} \underline{d}^{O_{S}}\left(X_{S}^{\nu}\right) \cong J_{X_{S}^{\nu}}$ and $\underline{d}^{O_{S}}$ is the multidegree associated to a totally cyclic orientation on $G-S$. Hence the boundary of the compactified degree- $(g-1)$ Jacobian of $X$ is stratified by Jacobians of degree $\left(g\left(X^{\prime}\right)-1\right)$ of partial normalizations, $X^{\prime}$, of $X$.

Remark 4.2.3. If $X$ is reducible and not of compact type then $\bar{P}_{X}^{g-1}$ is not of Néron type, by Remark 4.2.1(b).

A tropical counterpart of the stratification (11) is not known to us.

The compactified Jacobians in degree $g-1$ have been proved especially useful in various situations and in connection with the Theta divisor, whose definition extends to these compactified Jacobians. Among the applications, we recall that the pair given by this degree- $(g-1)$ Jacobian and its Theta divisor, $\left(\bar{P}_{X}^{g-1}, \bar{\Theta}_{X}\right)$, is endowed with a natural group action of $J_{X}$ and, as such, forms a so-called principally polarized stable semi-abelic pair. These pairs appear as boundary points in the compactification, $\bar{A}_{g}^{\text {mod }}$, of the moduli space of principally polarized abelian varieties constructed in [2]. Moreover, by [3, they form the image of the compactified Torelli map and we have

Proposition 4.2.4. The extended Torelli morphism $\bar{\tau}: \bar{M}_{g} \rightarrow \bar{A}_{g}^{\text {mod }}$ mapping a curve $X$ to $\left(\bar{P}_{X}^{g-1}, \bar{\Theta}_{X}\right)$ is the moduli map associated to the family $\psi_{g-1}: \bar{P}_{g}^{g-1} \rightarrow \bar{M}_{g}$.

The combinatorial structure of $\bar{P}_{X}^{g-1}$ described in Proposition 4.2 .2 is heavily used in 15 to study the fibers of $\bar{\tau}$.

4.3. Jacobians in degree $g$ : Néron compactified Jacobians. We now adapt the considerations at the beginning of the previous sections to the case $d=g$. We have $g=\sum_{v \in V}(h(v)-1)+|E|+1$ and, as before, we want to express the term $|E|+1$ in a combinatorially meaningful way. Modifying what we did earlier, we now consider orientations having one bioriented edge. More precisely, a 1-orientation on a graph $G$ is the datum of a bioriented edge, $e$, and of an orientation on $G-e$. For any 1-orientation we have, of course, $\left|\underline{d}^{O}\right|=g(G)$. Just as in the previous subsection we restricted to totally cyclic orientations, now we need to restrict to certain special 1orientations, namely "rooted" orientations. A 1-orientation with bioriented edge $e$ is said to be rooted (or e-rooted) if for every vertex $v$ there exists a directed path from $e$ to $v$.

As before, two 1-orientations $O$ and $O^{\prime}$ such that $\underline{d}^{O}=\underline{d}^{O^{\prime}}$ are defined to be equivalent, moreover one is rooted if the other one is. We denote by $\bar{O}^{1}(G)$ the set of equivalence classes of rooted 1-orientations on $G$.

We decree the empty orientation on the graph consisting of only vertex and no edges to be rooted. Similarly to Remark 4.2.1 we have:

Remark 4.3.1. (a) $\bar{O}^{1}(G)$ is not empty if and only if $G$ is connected. 
(b) $\left|\bar{O}^{1}(G)\right|=\left|\Phi_{G}\right|$.

In the picture below we have all $e$-rooted orientations on a 4-cycle with fixed bioriented edge $e$. They correspond to the four elements in $\bar{O}^{1}(G)$.
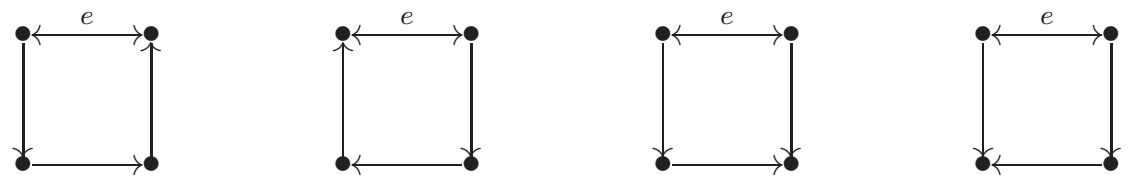

In Theorem 2.3.1 we exhibited a stratification indexed by the poset of connected spanning subgraphs, $\mathcal{C}(G)$. The strata of that stratification are not connected, we shall now exhibit a finer stratification with connected strata.

Recall that rooted 1-orientations exist only on connected graphs. The poset of equivalence classes of rooted 1-orientations on all spanning subgraphs of $G$ is written as follows

$$
\overline{\mathcal{O P}}^{1}(G):=\bigsqcup_{S \in \mathcal{C}(G)} \bar{O}^{1}(G-S),
$$

with the partial order defined in the previous section.

The following result of [17] states that $\bar{P}_{X}^{g}$ admits a recursive graded stratification governed by rooted orientations.

Theorem 4.3.2 (Christ). Let $X$ be a stable curve of genus $g$ and $G$ its dual graph. Then $\bar{P}_{X}^{g}$ admits the following graded stratification

$$
\bar{P}_{X}^{g}=\underset{\bar{O}_{S} \in \overline{\mathcal{O P}}^{1}(G)}{ } P_{X}^{O_{S}},
$$

and we have natural isomorphisms for every $\overline{O_{S}} \in \overline{\mathcal{O P}}^{1}(G)$

$$
P_{X}^{O_{S}} \cong \operatorname{Pic}^{\underline{d}^{O}}\left(X_{S}^{\nu}\right)
$$

Remark 4.3.3. Comparing with the stratification of Theorem 2.3.1 we have, using (13), that (14) is a refinement of (7), with connected strata.

In this case we do have a tropical counterpart. First, consistently with the dual stratifications (9) and (10), a tropical counterpart of $X$ is a tropical curve $\Gamma$ whose underlying graph is the dual graph of $X$.

We now assume $\Gamma=(G, \ell)$ is compact (i.e. not extended). The tropical curve $\Gamma$ has a Picard group $\operatorname{Pic}(\Gamma)=\sqcup \operatorname{Pic}^{d}(\Gamma)$, and each connected component, $\operatorname{Pic}^{d}(\Gamma)$, is isomorphic to the same $b_{1}(G)$-dimensional real torus; see 28. In [4, An-Baker-Kuperberg-Shokrieh show that $\operatorname{Pic}^{g}(\Gamma)$ has an interesting polyhedral decomposition indexed by "break divisors" on $G$. The connection between break divisors and rooted 1-orientations is established, 
as a consequence of the results in [4, in [17, where the following result is obtained.

Theorem 4.3.4. Let $\Gamma=(G, \ell)$ be a compact tropical curve of genus $g$. Then $\operatorname{Pic}^{g}(\Gamma)$ admits the following graded stratification

$$
\operatorname{Pic}^{g}(\Gamma)=\underset{\bar{O}_{S} \in \overline{\mathcal{O P}}^{1}(G)^{*}}{ } \Sigma_{\Gamma}^{O_{S}}
$$

The stratification (16) is a non-trivial rephrasing of the polyhedral decomposition established in [4]. Such a rephrasing is needed to establish the connection with the stratification (14). From [4] it follows that the strata $\Sigma_{\Gamma}^{O_{S}}$ are the interiors of the faces of a polyhedral decomposition for $\operatorname{Pic}^{g}(\Gamma)$.

Presently, we do not know whether the duality between the stratifications (16) and (14) can be given an interpretation in terms of tropicalization and analytification, similarly to the cases described in subsections 3.2 and 3.3 .

This problem is related to a result of [7, which we will state in our notation. Using Theorem 3.2.1, let $\mathcal{X}_{K}$ be a smooth curve, $J_{\mathcal{X}_{K}}$ its Jacobian, and let $\Gamma=\operatorname{trop}\left(\left[\mathcal{X}_{K}\right]\right)$, where $\left[\mathcal{X}_{K}\right]$ is the point of $\bar{M}_{g}^{\text {an }}$ corresponding to $\mathcal{X}_{K}$. Hence $\Gamma$ is a compact tropical curve of genus $g$ (compactness follows from $\mathcal{X}_{K}$ being smooth). With this set-up, [7, Thm. 1.3] yields

Theorem 4.3.5 (Baker-Rabinoff). $\operatorname{Pic}^{g}(\Gamma) \cong \Sigma\left(J_{\mathcal{X}_{K}}^{\text {an }}\right)$.

With this result in mind, a natural approach to the problem mentioned above would be to study the relation between $\Sigma\left(J_{\mathcal{X}_{K}}^{\text {an }}\right)$ and $\bar{P}_{X}^{g}$.

Finally, consider the universal compactified Jacobian. Results from [17] indicate that an analogue of Theorem 4.3.2 should hold uniformly over $\frac{\overline{\mathcal{M}_{g}}}{\text { }}$, so that the universal compactified Jacobian $\overline{\mathcal{P}}_{g}^{g}$ can be given a graded stratification compatible with that of $\overline{\mathcal{M}_{g}}$. We expect the same to hold for the universal compactified Jacobian in degree $g-1$, with Proposition 4.2 .2 as starting point.

\section{REFERENCES}

[1] Dan Abramovich, Lucia Caporaso, and Sam Payne. The tropicalization of the moduli space of curves. Ann. Sci. Éc. Norm. Supér. (4), 48(4):765-809, 2015.

[2] Valery Alexeev. Complete moduli in the presence of semiabelian group action. Ann. of Math. (2), 155(3):611-708, 2002.

[3] Valery Alexeev. Compactified Jacobians and Torelli map. Publ. Res. Inst. Math. Sci., 40(4):1241-1265, 2004.

[4] Yang An, Matthew Baker, Greg Kuperberg, and Farbod Shokrieh. Canonical representatives for divisor classes on tropical curves and the matrix-tree theorem. Forum Math. Sigma, 2:e24, 25, 2014.

[5] Matthew Baker, Sam Payne, and Joseph Rabinoff. On the structure of nonArchimedean analytic curves. In Tropical and non-Archimedean geometry, volume 605 of Contemp. Math., pages 93-121. Amer. Math. Soc., Providence, RI, 2013.

[6] Matthew Baker, Sam Payne, and Joseph Rabinoff. Nonarchimedean geometry, tropicalization, and metrics on curves. Algebr. Geom., 3(1):63-105, 2016. 
[7] Matthew Baker and Joseph Rabinoff. The skeleton of the Jacobian, the Jacobian of the skeleton, and lifting meromorphic functions from tropical to algebraic curves. Int. Math. Res. Not. IMRN, (16):7436-7472, 2015.

[8] Arnaud Beauville. Prym varieties and the Schottky problem. Invent. Math., 41(2):149-196, 1977.

[9] Vladimir Berkovich. Spectral theory and analytic geometry over non-Archimedean fields, volume 33 of Mathematical Surveys and Monographs. American Mathematical Society, Providence, RI, 1990.

[10] Silvia Brannetti, Margarida Melo, and Filippo Viviani. On the tropical Torelli map. Adv. Math., 226(3):2546-2586, 2011.

[11] Lucia Caporaso. A compactification of the universal Picard variety over the moduli space of stable curves. J. Amer. Math. Soc., 7(3):589-660, 1994.

[12] Lucia Caporaso. Néron models and compactified Picard schemes over the moduli stack of stable curves. Amer. J. Math., 130(1):1-47, 2008.

[13] Lucia Caporaso. Algebraic and tropical curves: comparing their moduli spaces. In Handbook of Moduli, Volume I, volume XXIV of Advanced Lectures in Mathematics, pages 119-160. International Press, Boston, 2012.

[14] Lucia Caporaso. Compactified Jacobians of Néron type. Atti Accad. Naz. Lincei Rend. Lincei Mat. Appl., 23(2):213-227, 2012.

[15] Lucia Caporaso and Filippo Viviani. Torelli theorem for graphs and tropical curves. Duke Math. J., 153(1):129-171, 2010.

[16] Renzo Cavalieri, Hannah Markwig, and Dhruv Ranganathan. Tropicalizing the space of admissible covers. Math. Ann., 364(3-4):1275-1313, 2016.

[17] Karl Christ. Orientations, break Divisors and compactified Jacobians. PhD thesis in Mathematics, Roma Tre University, 2017.

[18] Pierre Deligne and David Mumford. The irreducibility of the space of curves of given genus. Inst. Hautes Études Sci. Publ. Math., (36):75-109, 1969.

[19] Eduardo Esteves. Compactifying the relative Jacobian over families of reduced curves. Trans. Amer. Math. Soc., 353(8):3045-3095 (electronic), 2001.

[20] David Gieseker. Lectures on moduli of curves, volume 69 of Tata Institute of Fundamental Research Lectures on Mathematics and Physics. Published for the Tata Institute of Fundamental Research, Bombay; Springer-Verlag, Berlin-New York, 1982.

[21] Walter Gubler, Joseph Rabinoff, and Annette Werner. Skeletons and tropicalizations. Adv. Math., 294:150-215, 2016.

[22] Finn F. Knudsen. The projectivity of the moduli space of stable curves. II. The stacks $M_{g, n}$. Math. Scand., 52(2):161-199, 1983.

[23] Finn F. Knudsen. The projectivity of the moduli space of stable curves. III. The line bundles on $M_{g, n}$, and a proof of the projectivity of $\bar{M}_{g, n}$ in characteristic 0. Math. Scand., 52(2):200-212, 1983.

[24] Diane Maclagan and Bernd Sturmfels. Introduction to tropical geometry, volume 161 of Graduate Studies in Mathematics. American Mathematical Society, Providence, RI, 2015.

[25] Margarida Melo, Antonio Rapagnetta, and Filippo Viviani. Fine compactified Jacobians of reduced curves. Trans. Amer. Math. Soc., 369(8):5341-5402, 2017.

[26] Margarida Melo and Filippo Viviani. Fine compactified Jacobians. Math. Nachr., 285(8-9):997-1031, 2012.

[27] Grigory Mikhalkin. Moduli spaces of rational tropical curves. In Proceedings of Gökova Geometry-Topology Conference 2006, pages 39-51. Gökova Geometry/Topology Conference (GGT), Gökova, 2007.

[28] Grigory Mikhalkin and Ilia Zharkov. Tropical curves, their Jacobians and theta functions. In Curves and abelian varieties, volume 465 of Contemp. Math., pages 203-230. Amer. Math. Soc., Providence, RI, 2008. 
RECURSIVE COMBINATORIAL ASPECTS OF COMPACTIFIED MODULI SPACES 17

[29] André Néron. Modèles minimaux des variétés abéliennes sur les corps locaux et globaux. Inst. Hautes Études Sci. Publ.Math. No., 21:128, 1964.

[30] Tadao Oda and C. S. Seshadri. Compactifications of the generalized Jacobian variety. Trans. Amer. Math. Soc., 253:1-90, 1979.

[31] S. Payne. Analytification is the limit of all tropicalizations. Math. Res. Lett., 16(3):543-556, 2009.

[32] Michel Raynaud. Spécialisation du foncteur de Picard. Inst. Hautes Études Sci. Publ. Math., (38):27-76, 1970.

[33] Amaury Thuillier. Géométrie toroïdale et géométrie analytique non archimédienne. Application au type d'homotopie de certains schémas formels. Manuscripta Math., 123(4):381-451, 2007.

[34] Ilya Tyomkin. Tropical geometry and correspondence theorems via toric stacks. Math. Ann., 353(3):945-995, 2012.

[35] Filippo Viviani. Tropicalizing vs. compactifying the Torelli morphism. In Tropical and non-Archimedean geometry, volume 605 of Contemp. Math., pages 181-210. Amer. Math. Soc., Providence, RI, 2013.

(Caporaso) Dipartimento di Matematica e Fisica, Università Roma Tre, Largo San Leonardo Murialdo, I-00146 Roma, Italy

E-mail address: caporaso@mat.uniroma3.it 